\title{
Determinantes sociales de la salud asociados al grado de discapacidad en la ciudad de Barranquilla
}

\section{Social determinants of health associated with the degree of disability in the city of Barranquilla}

\author{
María Henríquez-Thorrens ${ }^{1}$ (D) , Anabell Donado-Mercado² (D), Tatiana Lían-Romero ${ }^{3}$ (iD) , José Armando Vidarte-Claros ${ }^{4}$ (iD \\ Consuelo Vélez-Álvarez ${ }^{5}$ (iD)
}

\begin{abstract}
Universidad Simón Bolívar. Barranquilla, Colombia. Correo: mhenriquez@unisimonbolivar.edu.co - https://orcid.org/0000-0003-4779-1674 Universidad Simón Bolívar. Barranquilla, Colombia. Correo: adonado1@unisimonbolivar.edu.co - https://orcid.org/0000-0003-3552-144XE Universidad Simón Bolívar. Barranquilla, Colombia. Correo: tlian@unisimonbolivar.edu.co -https://orcid.org/0000-0003-2010-3552 Universidad Autónoma de Manizales. Manizales, Colombia. Correo: jovida@autonoma.edu.co - https://orcid.org/0000-0002-7982-3848 Universidad Autónoma de Manizales. Manizales, Colombia. Correo: cva@autonoma.edu.co - https://orcid.org/0000-0001-7274-7304
\end{abstract}

Tipología: Artículo de investigación científica y tecnológica

Para citar este artículo: Henríquez-Thorrens M, Donado-Mercado A, Lían-Romero T, Vidarte-Claros A, Vélez-Álvarez C. Determinantes sociales de la salud asociados al grado de discapacidad en la ciudad de Barranquilla. Duazary. 2020 enero; 17(1): 49 - 61. Doi: http://dx.doi.org/10.21676/2389783X.3221

Recibido en febrero 01 de 2019

Aceptado en julio 01 de 2019

Publicado en línea en noviembre 19 de 2019

\section{RESUMEN}

Palabras

clave:

Discapacidad;

empleo;

escolaridad, determinantes

sociales de la

salud; clase

social;

rehabilitación.
El objetivo de esta investigación fue establecer la asociación de los determinantes sociales de la salud (DSS) con el grado de discapacidad en población mayor de 18 años de la ciudad de Barranquilla. Se realizó un estudio descriptivo, con 216 personas con discapacidad. Como variable dependiente se midió el grado de discapacidad con el WHODAS 2.0, y como variables independientes se evaluaron los determinantes sociales de la salud. El 56,9\% fueron mujeres, y la edad promedio fue de 53,68 años. De acuerdo con el grado de discapacidad, el mayor porcentaje se encontró, en el grado extremo, en el dominio actividades de la vida diaria-trabajo remunerado y, en el grado moderado, en los dominios de participación en la sociedad. A medida que aumentan la edad, las horas de trabajo, el tiempo de trabajo y los años sin rehabilitación se ven afectados los dominios de comprensión y comunicación, movilidad, autocuidado, relaciones con otras personas, actividades de la vida diaria-trabajo no remunerado, actividades de la vida diaria-trabajo remunerado, participación en sociedad y con el grado discapacidad final y que esta asociación es estadísticamente significativa $p<0,05$. Se concluye que existe asociación entre los determinantes sociales de la salud y el grado de discapacidad en la población estudiada.

\section{ABSTRACT}

Keywords:

Disability; employment;

level of education; social determinants of health; social class; rehabilitation.
The objective of this study was to establish the association of the Social Determinants of Health (DSS) with the degree of disability in a population over 18 years of age in the city of Barranquilla. A descriptive study was carried out, with 216 people with disabilities. As a dependent variable, the degree of disability was measured with the WHODAS 2.0 and the social determinants of health were evaluated as independent variables. $56.9 \%$ were women, the average age was 53.68 years, according to the degree of disability the highest percentage was found in the extreme degree the activities of daily living-paid work and in the moderate degree the domains of participation in society. As the age increases, the hours of work, the time of work and the years without rehabilitation are affected the domains of understanding and communication, mobility, self-care, relationships with other people, activities of daily life-unpaid work, activities of daily living-paid work, participation in society and with the final disability degree and that this association is statistically significant $p<0.05$. It is concluded that there is an association between the social determinants of health and the degree of disability in the population studied. 


\section{INTRODUCCIÓN}

Las inequidades sociales son generadoras de vida o muerte, salud o enfermedad, riqueza o pobreza. El hecho de que en una misma ciudad la población esté expuesta a diferentes circunstancias sociales ${ }^{1}$ trae como consecuencia desigualdades a nivel de salud, de oportunidades, de ingresos, de educación y de otros indicadores de logro social, convirtiéndose en una influencia negativa en el desarrollo humano ${ }^{2}$.

El análisis desde la perspectiva de los determinantes sociales de la salud (DSS) aparece como una nueva manera de entender y explicar las inequidades en salud. Los DSS son definidos como las "circunstancias donde las personas nacen, crecen, viven, trabajan y envejecen", y se ven influenciados por diferentes aspectos como los recursos, el mercado laboral, la distribución del dinero, entre otros, en los diferentes contextos internacionales, nacionales y locales y tienen relación directa con las políticas adoptadas en estos ${ }^{2}$.

Los DSS pueden ser explicados por diferentes modelos. En este artículo se parte del modelo conceptual adoptado por el Ministerio de Salud y Protección Social, postulado por la Organización Mundial de la Salud (Modelo Solar e Irvin). Este busca determinar aquellos elementos de la estructura social que condicionan la posición de las personas dentro de la sociedad ya que tienen un efecto directo en la distribución de los problemas o de los factores protectores de la salud. De este modo se muestra la interrelación de los aspectos de contexto, posición y circunstancias con la equidad sanitaria ${ }^{3}$.

Los DSS se clasifican en estructurales -es decir, aquellas condiciones del macrocontexto que implican niveles jerárquicos, condicionan la estructura social y se asocian de manera estrecha con el poder, la reputación y los recursos (esta interacción y la manera como se presenta en los sujetos es generadora de desigualdades sociales)-, los cuales influyen de manera directa ${ }^{4}$, e intermedios - asumidos como aquellas condiciones cercanas a los sujetos, entre las cuales se encuentran las condiciones de vida y trabajo, la disponibilidad de alimentos, las conductas y los factores biológicos (hábitos de consumo y estilos de vida) y los factores psicosociales ${ }^{2}-$. En particular, se resalta que el sistema de salud se considera como un determinante intermedio, ya que el sector sanitario ejerce influencia en la producción de la salud, con responsabilidad en la gestión de las diferencias en la vulnerabilidad, en la exposición y en las consecuencias de la enfermedad ${ }^{4,5}$.

En Colombia, el Observatorio Nacional de Salud (ONS) pretende profundizar en el análisis de la situación de salud en el país con la perspectiva de DSS. Para ello examina problemas prioritarios en salud o la situación de salud de grupos específicos en territorios determinados, con miras a entender los determinantes y brindar elementos para abordarlos a través de políticas públicas. De este modo se espera contribuir a la mejoría de condiciones de salud de la población del país y disminuir las inequidades en la materia ${ }^{6}$.

De otro lado, la discapacidad, asumida desde lo multidimensional, es considerada una situación heterogénea. El nuevo enfoque, dado por la Organización Mundial de la Salud (OMS), se centra en el modo en que los componentes del funcionamiento humano inciden en la interacción de la persona con su estado de salud. Factores personales, como edad, género, etnia y nivel educativo, y factores contextuales demuestran la existencia de un hilo conector decisivo entre la dinámica de la formación social y la desigualdad y vulnerabilidad social que experimenta la persona con discapacidad y que la predispone a vivir en condición de pobreza. Adicionalmente, este grupo poblacional es el que más sufre discriminación y exclusión social ${ }^{7}$. Todo esto conlleva a abordar la discapacidad como constructo genérico, multifactorial y sistémico.

La discapacidad incluye problemas en el funcionamiento o en las estructuras del cuerpo que pueden generar desde limitaciones en las actividades de la vida diaria hasta restricciones en la participación. Todos los seres humanos están expuestos a tener una discapacidad, ya sea en 
menor o mayor grado, de forma transitoria o permanente, $y$ en algunos casos se pueden generar incapacidades en la realización de ciertas labores. Se puede afirmar que los grados de discapacidad serán diferentes siempre y cuando exista rehabilitación, y según se viva en área rural o urbana o en un país desarrollado o en vía de desarrollo ${ }^{8}$.

La clasificación de la discapacidad ha sido muy diversa. En la 54 Asamblea Mundial de la Salud de la OMS, se cambió el nombre de la segunda edición de la Clasificación Internacional de las Deficiencias, Discapacidades y Minusvalías (CIDDM-2) por Clasificación Internacional del Funcionamiento, de la Discapacidad y de la Salud (CIF), que ha sido considerada un instrumento estándar para medir la salud y la discapacidad tanto individual como poblacional. Esta clasificación se basa en la salud y en dominios relacionados con esta desde la perspectiva del cuerpo, el individuo y la sociedad (biopsicosocial) $^{9,10}$.

En búsqueda de establecer relaciones entre DSS y la discapacidad, se han realizado diferentes estudios que han abordado elementos de inclusión social familiar y comunitaria, la participación social de personas con deficiencia, la inserción en ocupaciones en la vida cotidiana, el vínculo bidireccional con la pobreza, la relación entre discapacidad y el riesgo de pobreza, y viceversa ${ }^{11-13}$. Así mismo, el estudio del Comité Español de Representantes de Personas con Discapacidad (CERMI) sobre la discapacidad y exclusión social en la Unión Europea se refiere a la falta de acceso al entorno social y a los servicios o la limitación de este, el desempleo, la estigmatización de las personas discapacitadas, la falta de formación adecuada y el impacto de la discapacidad sobre los padres (la mayor parte madres) de personas con discapacidad severa, que deben compensar esta falta en detrimento de su trabajo, ingresos y derechos de pensión, así como de su propia participación en la comunidad y el entorno social ${ }^{14-}$ 16

El objetivo de este trabajo fue establecer los determinantes sociales de la salud asociados con el grado de discapacidad en población mayor de 18 años de la ciudad de Barranquilla. El conocimiento de la relación que cada uno de los determinantes sociales de la salud analizados tiene con el grado de discapacidad constituye un insumo importante para la propuesta e implementación de políticas públicas que propendan a adecuados procesos integrales y de inclusión social para esta población.

\section{MATERIALES Y MÉTODOS}

\section{Tipo de estudio}

Se realizó un estudio de tipo cuantitativo, descriptivo.

\section{Población y muestra}

Población: personas con discapacidad identificadas a través del Registro de Caracterización y Localización de Personas con Discapacidad de la Ciudad Barranquilla, mayores de 18 años de edad.

Muestra: tal como se presenta en la tabla 1, con un nivel de confianza del $95 \%$ se estimó una muestra de 194 personas y se asumió un porcentaje de pérdida del $10 \%$. Finalmente, la muestra calculada fue de 216 personas. Se utilizó para el cálculo la siguiente fórmula:

\section{Cálculo del tamaño de la muestra para estimar una correlación lineal \\ Test bilateral:}

$$
n=\left(\frac{z_{1}-\alpha / 2+z_{1}-\beta}{\frac{1}{2} 1 n\left(\frac{1+r}{1-r}\right)}\right)^{2}+3
$$


Tabla 1. Estimadores y valores para el cálculo de la muestra.

\begin{tabular}{|c|c|c|}
\hline Estimadores & Valores & Valores \\
\hline Nivel de confianza $(Z$ alfa): $95 \%$ & 1,96 & 1,96 \\
\hline Poder estadístico $(Z$ beta): $80 \%$ & 0,842 & 0,842 \\
\hline Correlación lineal esperada $(r)$ & 0,30 & 0,20 \\
\hline Tamaño de la muestra $(n)$ & 85 & 194 \\
\hline Porcentaje de pérdida $(\mathrm{L})$ & 0,10 & 0,10 \\
\hline Muestra ajustada a la perdida $\left(\mathrm{n}^{\prime}\right)$ & 94 & 216 \\
\hline
\end{tabular}

\section{Técnicas e instrumentos}

Se utilizó la encuesta como técnica, con un formato previamente elaborado por los investigadores para evaluar los DSS. Para evaluar el grado de discapacidad según los diferentes dominios, se aplicó el WHODAS 2.0 con 36 ítems, el cual es conceptualmente compatible con el modelo de la CIF.

\section{Procedimiento}

Para obtener la información de la población se realizó contacto con la Secretaría de Salud Distrital y con entidades que trabajan con discapacidad. Esta recolección de datos posibilitó la calibración de los evaluadores, que para este caso fueron los investigadores. Así mismo, se reclutaron a los participantes del estudio según la muestra calculada y, previo a la aplicación de los instrumentos, se obtuvo la aceptación y firma del consentimiento informado.

Para el análisis estadístico se utilizó el programa SPSS versión 24.0, licenciado por la Universidad Autónoma de Manizales. Se calcularon las medidas de tendencia central y de variabilidad o dispersión para variables cuantitativas, mientras que las variables cualitativas se analizaron a través de distribución de frecuencias absolutas y relativas.

Para el análisis bivariado se aplicaron las pruebas establecidas a partir de las características propias de las variables. En la correlación se utilizó el coeficiente de Spearman para las variables de comportamiento no normales y el de Pearson para las normales. Se consideró la significancia estadística cuando $p \leq 0,05$. Además, se tuvo en cuenta lo establecido por Mukaka ${ }^{17}$, de manera que un coeficiente de correlación de 0,2 o menos se asumió como una correlación insignificante. Para las variables cualitativas se realizaron medidas de asociación con tablas de contingencia utilizando el estadístico Chi cuadrado.

\section{Declaración sobre aspectos éticos}

La investigación cumplió con los principios éticos de la Declaración de Helsinki. Así mismo, de acuerdo a la Resolución 008430, artículo 11, el presente estudio se consideró como "investigación con riesgo mínimo" debido a la no manipulación de variables biológicas. Para su realización, se diligenció el consentimiento informado por parte de los participantes, o de su representante legal, mediante el cual se brindó toda la información acerca de los riesgos y los beneficios del estudio. Los participantes tuvieron plena autonomía para aceptar o rechazar la participación en el trabajo. El proyecto fue aprobado por el comité de bioética de la Universidad Autónoma de Manizales.

\section{RESULTADOS}

Con respecto a la distribución de los DSS estructurales e intermedios en la ciudad de Barranquilla, se evidenció que prevalecieron el sexo femenino $(56,1 \%)$, la etnia mestiza $(97,2 \%)$, los estratos socioeconómicos 1 y 2 (57,8\%), edades menores de 45 años $(38,4 \%)$, el estado civil casado $(35,6 \%)$, la formación en algún tipo de educación básica $(58,4 \%)$, la afiliación al Sistema General de Seguridad Social en Salud (100\%), la falta de ingresos económicos $(54,4 \%)$ y de pensión por discapacidad $(91,7 \%)$, y personas que viven en casa $(77,8 \%)$. Un bajo porcentaje $(35,2 \%)$ recibe servicios de rehabilitación, y un 60,2\% atribuye su recuperación a los servicios de salud y servicios prestados. Por su 
parte, el tipo de rehabilitación más ordenada fue fisioterapia (30,1\%), y el 39,4\% terminó rehabilitación y lleva entre 11 y 60 años sin ella. En cuanto al grado de discapacidad, el mayor porcentaje se encontraba en grado moderado, seguido del leve (tabla 2).

Tabla 2. Estadísticos descriptivos del grado de discapacidad final WHODAS 2.0.

\begin{tabular}{|c|c|c|c|}
\hline Dominio & Grado discapacidad & $\#$ & \% \\
\hline Cognición & Leve & 75 & 34,7 \\
\hline Movilidad & Severa & 75 & 34,7 \\
\hline Cuidado personal & Moderada & 76 & 35,2 \\
\hline Relaciones & Leve & 108 & 50 \\
\hline Trabajo no remunerado & Moderado & 100 & 46,3 \\
\hline Trabajo remunerado & Extrema & 127 & $\mathbf{5 8 , 8}$ \\
\hline Participación & Moderada & 116 & 53,7 \\
\hline
\end{tabular}

En la tabla 3 se presenta el análisis descriptivo de la puntuación por dominios. Nótese cómo el dominio con la media de mayor puntuación fue actividades de la vida diaria, y el de menor fue comprensión y comunicación. De igual forma, es de resaltar cómo el dominio capacidad de moverse en el entorno también presenta una puntuación alta. Con respecto a la desviación estándar, la más alta encontrada fue la de actividades de la vida diaria, lo que sugiere heterogeneidad de los datos en este dominio entre las diferentes personas (tabla 3 ).

Tabla 3. Estadísticos descriptivos de la puntuación final y por dominios del WHODAS 2.0.

\begin{tabular}{|c|c|c|c|c|}
\hline Dominios & Mínimo & Máximo & Media & Desv. Típ. \\
\hline Comprensión y comunicación (Do1) & 0,00 & 100,00 & 12,6852 & 17,17795 \\
\hline Capacidad de moverse en su entorno ( Do2) & 0,00 & 100,00 & 44,5891 & 28,05824 \\
\hline Cuidado personal (Do3) & 0,00 & 100,00 & 24,7222 & 24,28353 \\
\hline Relacionarse con otras personas (Do4) & 0,00 & 91,67 & 13,6574 & 17,23670 \\
\hline $\begin{array}{c}\text { Actividades de la vida diaria } \\
\text { (Do5.1) }\end{array}$ & 0,00 & 100,00 & 50,3241 & 32,38413 \\
\hline Actividades de la vida diaria-trabajo remunerado (Do5.2) & 0,00 & 71,43 & 19,9037 & 16,76781 \\
\hline $\begin{array}{c}\text { Participación en sociedad } \\
\text { (Do6) }\end{array}$ & 0,00 & 95,83 & 39,3711 & 17,13358 \\
\hline Discapacidad final WHODA 2.0 (st_s32) & 1,09 & 96,74 & 30,7216 & 16,04358 \\
\hline
\end{tabular}

Se evidenció una relación estadísticamente significativa $(p<0,05)$ entre la discapacidad final y la capacidad de moverse en su entorno con la edad. Los determinantes estructurales de la salud ingreso mensual, horas de trabajo, tiempo de trabajo y años sin rehabilitación presentaron una relación estadísticamente significativa e inversa con actividades de la vida diaria. Así mismo, en horas de trabajo y tiempo de trabajo se observa una relación estadísticamente significativa e inversa con participación en sociedad. Por otro lado, las horas de trabajo tienen una relación estadísticamente significativa e inversa con discapacidad final WHODAS 2.0 (tabla 4). 
Tabla 4. Correlación entre los determinantes sociales de la salud estructurales e intermedios con el nivel de discapacidad por dominios y final (WHODAS 2.0).

\begin{tabular}{|c|c|c|c|c|c|c|c|c|c|}
\hline Variables & Estadístico & Do1 & Do2 & Do3 & Do4 & Do51 & Do52 & Do6 & St-s32 \\
\hline \multirow{4}{*}{ Edad } & Spearman & 0,21 & & 0,08 & 0,20 & 0,27 & 0,06 & & \\
\hline & P valor & $0,00 *$ & & 0,36 & $0,01^{*}$ & $0,00 *$ & 0,88 & & \\
\hline & Pearson & & 0,32 & & & & & 0,18 & 0,32 \\
\hline & P valor & & $0,00^{*}$ & & & & & $0,00 *$ & $0,00 *$ \\
\hline \multirow{4}{*}{ Ingreso mensual } & Spearman & 0,80 & & $-0,08$ & $-0,31$ & $-0,30$ & $-0,10$ & & \\
\hline & P valor & 0,24 & & $0,00^{*}$ & $0,00 *$ & $0,00 *$ & 0,33 & & \\
\hline & Pearson & & $-0,09$ & & & & & $-0,19$ & $-0,20$ \\
\hline & $\mathrm{P}$ valor & & 0,18 & & & & & $0,00 *$ & $0,00 *$ \\
\hline \multirow{4}{*}{ Horas de trabajo } & Spearman & $-0,91$ & & $-0,20$ & $-0,24$ & $-0,33$ & $-0,38$ & & \\
\hline & P valor & $0,00^{*}$ & & $0,00^{*}$ & $0,00 *$ & $0,00 *$ & 0,52 & & \\
\hline & Pearson & & $-0,26$ & & & & & $-0,31$ & $-0,37$ \\
\hline & $\mathrm{P}$ valor & & $0,00 *$ & & & & & $0,00 *$ & $0,00 *$ \\
\hline \multirow{4}{*}{ Tiempo de trabajo } & Spearman & $-0,23$ & & $-0,19$ & $-0,30$ & $-0,36$ & $-0,41$ & & \\
\hline & P valor & $0,00^{*}$ & & $0,00^{*}$ & $0,00 *$ & $0,00 *$ & $0,00 *$ & & \\
\hline & Pearson & & $-0,15$ & & & & & $-0,38$ & $-0,21$ \\
\hline & $\mathrm{P}$ valor & & 0,25 & & & & & $0,00 *$ & $0,00 *$ \\
\hline \multirow{4}{*}{$\begin{array}{c}\text { Número de personas } \\
\text { con quien comparte la } \\
\text { vivienda }\end{array}$} & Spearman & $-0,04$ & & $-0,09$ & 0,07 & 0,00 & 0,01 & & \\
\hline & P valor & 0,51 & & 0,18 & 0,25 & 0,99 & 0,82 & & \\
\hline & Pearson & & $-0,08$ & & & & & 0,00 & $-0,03$ \\
\hline & P valor & & 0,22 & & & & & 0,96 & 0,62 \\
\hline \multirow{4}{*}{ Años sin rehabilitación } & Spearman & $-0,11$ & & $-0,18$ & $-0,16$ & $-0,31$ & $-0,29$ & & \\
\hline & P valor & 0,09 & & $0,00^{*}$ & $0,01^{*}$ & $0,00 *$ & $0,00 *$ & & \\
\hline & Pearson & & $-0,18$ & & & & & $-0,17$ & $-0,16$ \\
\hline & P valor & & $0,00 *$ & & & & & $0,01^{*}$ & $0,01^{*}$ \\
\hline
\end{tabular}

$P$ valor: significancia estadística bilateral.

*Correlación significativa al nivel 0,05 (bilateral).

Se encontró asociación estadísticamente significativa $(p<0,05)$ entre el grado de discapacidad y los DSS estructurales edad y nivel educativo, así como con los DSS intermedios ingreso, horas de trabajo, tiempo de trabajo, tipo de contrato de trabajo, condiciones de seguridad en el trabajo, niveles de ruido en el lugar de trabajo, exposición a temperaturas extremas (lluvia, calor), en qué se desplaza hasta su sitio de trabajo, qué actividad realizaba antes de la discapacidad, utiliza órtesis y/o prótesis, recibe servicios de rehabilitación, a qué factores atribuye su recuperación (servicios de salud y servicios recibidos), tipo de rehabilitación ordenada (terapia ocupacional) y medicamentos permanentes (tabla 5). 
Tabla 5. Asociación entre determinantes sociales de la salud estructurales e intermedios y grado de discapacidad.

\begin{tabular}{|c|c|c|}
\hline Determinantes sociales de la salud estructurales & Chi cuadrado & P valor \\
\hline 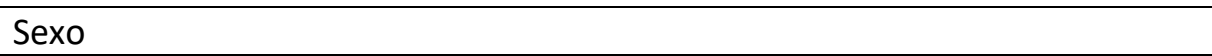 & 44,5 & 0,34 \\
\hline Edad & 24,5 & $0,00 *$ \\
\hline Estado civil & 29,8 & 0,07 \\
\hline Estrato social & 29,4 & 0,08 \\
\hline Nivel educativo & 81,7 & $0,00^{*}$ \\
\hline Etnia & 3,47 & 0,90 \\
\hline Determinantes sociales de la salud intermedios & Chi cuadrado & P valor \\
\hline Tipo de afiliación & 4,97 & 0,76 \\
\hline Ingreso & 20,4 & $0,00^{*}$ \\
\hline Recibe pensión por discapacidad & 2,68 & 0,61 \\
\hline Horas de trabajo & 28,2 & $0,00 *$ \\
\hline Tiempo de trabajo & 40,7 & $0,00^{*}$ \\
\hline Tipo de contrato & 27,9 & $0,00 *$ \\
\hline Condiciones de seguridad en el trabajo & 34,3 & $0,00 *$ \\
\hline Nivel del ruido & 34,4 & $0,00 *$ \\
\hline Exposición a temperaturas extremas & 14,5 & $0,00 *$ \\
\hline En qué se desplaza para ir al trabajo & 41,4 & $0,00 *$ \\
\hline Actividad que realizaba antes de la discapacidad & 41,1 & $0,00^{*}$ \\
\hline Utiliza órtesis y/o prótesis & 10,7 & $0,03^{*}$ \\
\hline En este momento usted habita en & 6,44 & 0,59 \\
\hline El inmueble que está habitando es & 4,65 & 0,79 \\
\hline Con quién vive actualmente: solo & 2,09 & 0,71 \\
\hline Con quién vive actualmente: pareja & 5,50 & 0,23 \\
\hline Con quién vive actualmente: papá & 1,15 & 0,88 \\
\hline Con quién vive actualmente: mamá & 0,83 & 0,93 \\
\hline Con quién vive actualmente: familiares & 15,0 & 0,05 \\
\hline Con quién vive actualmente: amigos & 0,57 & 0,96 \\
\hline Recibe rehabilitación & 12,9 & $0,01^{*}$ \\
\hline Factor a que atribuye su recuperación: servicios de salud y servicios recibidos & 11,2 & $0,02 *$ \\
\hline Factor a que atribuye su recuperación: apoyo familiar & 3,52 & 0,47 \\
\hline Factor a que atribuye su recuperación: empeño a salir adelante & 4,31 & 0,36 \\
\hline Factor a que atribuye su recuperación: a Dios & 6,92 & 0,14 \\
\hline Factor a que atribuye su recuperación: medicina alternativa & 0,57 & 0,96 \\
\hline Tipo de rehabilitación ordenada: medicina física y Rh & 1,90 & 0,57 \\
\hline Tipo de rehabilitación ordenada: psiquiatría & 6,19 & 0,18 \\
\hline Tipo de rehabilitación ordenada: fisioterapia & 10,0 & 0,39 \\
\hline Tipo de rehabilitación ordenada: fonoaudiología & 1,65 & 0,79 \\
\hline Tipo de rehabilitación ordenada: terapia ocupacional & 14,5 & $0,00^{*}$ \\
\hline Tipo de rehabilitación ordenada: optometría & 2,56 & 0,63 \\
\hline Tipo de rehabilitación ordenada: psicología & 9,35 & 0,05 \\
\hline Tipo de rehabilitación ordenada: trabajo social & 2,29 & 0,68 \\
\hline Tipo de rehabilitación ordenada: medicamentos permanentes & 14,5 & $0,00 *$ \\
\hline
\end{tabular}

P valor: significancia estadística bilateral.

*Asociación estadísticamente significativa al nivel 0,05 (bilateral). 


\section{DISCUSIÓN}

Esta investigación evidenció la asociación entre los DSS y el grado de discapacidad para Barranquilla. Se encontró que las mujeres en mayor porcentaje $(56,9 \%)$ tienen discapacidad, lo que coincide con lo reportado en el Informe Mundial de Salud realizado por la Organización Mundial de la Salud en $2011^{7}$. Lo mismo se evidencia en el informe sobre las personas con discapacidad en América Latina de la Cepal, con base en datos recogidos en los censos realizados en algunos países latinoamericanos durante los años 2000 en adelante ${ }^{18}$. En Colombia, particularmente, el Ministerio de Salud y de la Protección Social reportó que a corte de diciembre de 2013 más mujeres que hombres se habían inscrito en el Registro para la Localización y Caracterización de las Personas con Discapacidad ${ }^{19}$.

Con respecto a la edad, esta investigación permitió establecer que la población más comprometida con discapacidad es menor de 45 años. Este hallazgo difiere de la Encuesta de Discapacidad, Autonomía Personal y Situaciones de Dependencia (EDAD) ${ }^{20}$ realizada en España, donde la población comprometida es mayor de 45 años, así como del informe de la Cepal, que reportó que el porcentaje de discapacidad aumenta significativamente en el grupo de edades de mayores de 65 años ${ }^{14}$. Por otra parte, a nivel local, autores como Vélez y Vidarte ${ }^{16}$ en Colombia y Flores et al. ${ }^{21}$ en Honduras plantean un alto porcentaje de discapacidad en rangos de edad en el grupo de 30 a 34 años y 31 a 50 años respectivamente, datos que concuerdan con el presente estudio.

Al analizar el DSS estructural estrato social se evidencia una mayor proporción en los estratos 1 y 2 , con $24,5 \%$ y $33,3 \%$ respectivamente. Esto sigue la línea de autores como Gil et al. ${ }^{22}$ y Daza y Mosquera $^{23}$, quienes reportaron en sus estudios que el mayor porcentaje de población pertenece a estratos 2 y 1 respectivamente.

El estudio realizado por Fuentes et al. considera que los DSS desempeñan un papel importante en el proceso de discapacidad. Dentro de estos, el nivel socioeconómico ejerce una influencia directa en el proceso de pérdida funcional: se demostró que a más bajo nivel, mayor probabilidad de limitarse funcionalmente y menor posibilidad de recuperarse $\mathrm{e}^{24}$.

Con relación al nivel educativo, el 58,4\% de los participantes tienen algún tipo de educación básica. Este hallazgo es similar a otros encontrados a nivel nacional como el del Registro para la Localización y Caracterización de las Personas con Discapacidad del DANE (2010), según el cual el $55,3 \%$ de la población con discapacidad tiene algún tipo de educación básica ${ }^{25}$. Al respecto Castelblanco et al. ${ }^{26}$ encontraron que el $49,2 \%$ de la población del estudio había cursado básica primaria.

Lo anterior guarda relación con el análisis realizado por Samaniego ${ }^{27}$ acerca del acceso a la educación de las personas con discapacidad en América Latina. Según el autor, se ha visto que tradicionalmente los sistemas escolares agrupan a sus estudiantes según las características de los alumnos, buscando homogeneidad; a causa de esto, y bajo la premisa de dar una ayuda especial según las necesidades individuales de alumno, la población con discapacidad ha sido segregada. En la actualidad este paradigma ha venido cambiando de tal modo que se han aceptado las políticas de inclusión educativa, pero con más sombras que luces.

El derecho a los servicios de salud es un bien que todo ciudadano debe tener, independiente de su condición socioeconómica. En algunos países desarrollados, este bien es distribuido de acuerdo con la necesidad y no según la capacidad de pago del individuo, buscando la igualdad en la provisión de este derecho. En países como Canadá, la medicina privada solo sirve para acceder a ciertos servicios que no se consideran esenciales o son sencillos y no están cubiertos por los planes financiados con recursos públicos. De esta forma se logra una equidad en la prestación de los servicios de salud y se evita lo que sucede en algunos países que mantienen doble sistema de salud, uno público y otro privado, donde la balanza termina inclinándose hacia este último, atrayendo al mejor recurso humano y material del sistema de salud ${ }^{28}$. En cuanto al tipo de afiliación al Sistema General de Seguridad 
Social en Salud, esta investigación pudo determinar que el $100 \%$ de la población está afiliada a algún tipo de seguridad social, y el $68,1 \%$ pertenece al régimen contributivo.

La rehabilitación es concebida como la forma de prevención y control de los diferentes daños, enfocada a evitar alteraciones en la calidad de vida de los individuos que se encuentran en riesgo. A través de ella se espera recuperar las actividades perdidas $o$ aquellas que nunca antes se han practicado y de esta manera recobrar ciertas aptitudes físicas ${ }^{29}$. En el presente estudio se encontró que el $64,8 \%$ de la población participante no recibe servicios de esta clase, mientras que un menor porcentaje de la población sí los recibe. La rehabilitación más ordenada fue fisioterapia, con el $30,1 \%$.

Comparando este estudio con lo reportado por Vélez y Vidarte ${ }^{16}$, se encuentran diferencias con relación a la asistencia a rehabilitación: el $67,4 \%$ de la población investigada por dichos autores respondió que sí recibía servicios de rehabilitación, y el $32,6 \%$, que no. Daza y Mosquera ${ }^{23}$ por su parte encontraron que el tipo de rehabilitación más ordenada fue rehabilitación por fisioterapia. En todo caso, para la Organización Panamericana de la Salud, la atención de las personas con discapacidad es uno de los retos más importantes del sector salud, y en esa medida asegura que esta atención debe tener como propósito la prevención, la rehabilitación de la discapacidad, la mejora en la calidad de vida y la promoción de la vida independiente ${ }^{30}$.

La presente investigación evidenció que el $54,4 \%$ de la población no posee ningún ingreso y que el $91,7 \%$ no tiene pensión por discapacidad. A pesar de que existen leyes que fomentan la inclusión laboral, como la Ley 361 de 1997, la Ley 1346 del 2009, la Ley Estatutaria 1618 de 2013, entre otras, las cuales ofrecen beneficios para todas aquellas empresas que incluyan en su nómina a personas con discapacidad, sigue vulnerándose ese derecho. Los hallazgos en este estudio son similares al estudio nacional de Gil et al. ${ }^{22}$, que reportó que los participantes en su mayoría estaban desempleados por motivo de salud o realizaban trabajos informales o de forma independiente que evidenciaban inestabilidad. Esta problemática en la participación laboral también se evidencia a nivel internacional: Pupiales y Córdoba ${ }^{31}$, por ejemplo, reportaron que la tasa de desempleo en España es mayor en un 40\% en personas con discapacidad que en las que no tienen discapacidad y solo un $20 \%$ de la población contó con experiencia laboral.

Al analizar la discapacidad final, se encontró que el mayor porcentaje del grado de discapacidad se encuentra ubicado en la categoría moderada y leve, lo cual coincide con estudios internacionales en Chile y Honduras ${ }^{32,33}$, aunque a nivel nacional un estudio encontró que la población participante evidenció discapacidad leve. Si bien la discapacidad se relaciona con las dificultades a nivel de estructuras y funciones corporales, la atención oportuna de estas es fundamental en la disminución o en la ausencia de discapacidad ${ }^{34}$.

Así mismo, se encontró asociación entre los DSS edad, horas de trabajo, tiempo de trabajo y años sin rehabilitación y los dominios del WHODAS 2.0 comprensión y comunicación, movilidad, autocuidado, relaciones con otras personas, actividades de la vida diaria-trabajo no remunerado, actividades de la vida diaria-trabajo remunerado y discapacidad final. A su vez, no se observó asociación entre ingreso mensual promedio y movilidad, participación en sociedad y discapacidad final, ni entre el número de personas con quien se comparte la vivienda y los dominios del WHODAS 2.0. El estudio realizado por Gil et al. ${ }^{22}$ evidenció por otra parte una relación estadísticamente significativa entre la movilidad y las variables tipo de ayudas externas y tipo de medicamentos. Finalmente, los datos de ambos estudios concuerdan en la relación entre la mayoría de los determinantes sociales y el dominio actividades de la vida diaria y en la relación entre la edad y la discapacidad final.

También se encontró una asociación estadísticamente significativa entre el grado de discapacidad y los DSS intermedios órtesis y/o prótesis, recibe servicios de rehabilitación, a qué factores atribuye su recuperación servicios de salud y servicios recibidos, tipo de rehabilitación ordenada terapia ocupacional, y medicamentos permanentes. Esto lo han explicado algunos estudios que afirman 
que las personas con discapacidad experimentan obstáculos para el acceso a los servicios de salud y han evidenciado inequidades en la prestación de ellos y desequilibrio entre la oferta y la demanda, todo lo cual genera dificultades a la hora de garantizar una atención integral en salud, con estándares de calidad ${ }^{35}$.

Con respecto al DSS recibe servicio de rehabilitación y su asociación con el grado de discapacidad, no tener este servicio aumenta la probabilidad de mayor grado de discapacidad. En relación con los servicios sanitarios, la Organización Mundial de la Salud afirma que las personas con discapacidad tienen más demanda que las personas sin discapacidad y presentan más necesidades insatisfechas a este nivel, lo que puede traer como consecuencia una mayor vulnerabilidad a afecciones secundarias, comorbilidad, muerte prematura, entre otros ${ }^{36,37}$.

Sobre los hallazgos de este estudio que evidencian asociación entre los DSS y el grado de discapacidad, se sugiere generar cambios significativos a nivel familiar y social toda vez que las personas con discapacidad y sus familias a menudo no son conscientes de su potencial, de manera que se deben mejorar los procesos de orientación vocacional, inclusión educativa y laboral. El hecho de que un gran porcentaje de la población del estudio se encuentre ubicada en estrato socioeconómico bajo condiciona las posibilidades de desarrollar sus capacidades y de ser útil en su microsistema, mesosistema y macrosistema porque los determinantes sociales que la rodean se convierten en factores de riesgo para la discapacidad ${ }^{37,38}$.

En conclusión, se encontró que tanto los DSS estructurales como los intermedios tienen relación con el grado de discapacidad. Así pues, aspectos como no poder acceder a servicios de rehabilitación, las horas de trabajo, los ingresos económicos mensuales, entre otros, podrían reflejar que las políticas públicas en torno a la discapacidad son poco eficientes, lo que hace evidente la inequidad social y la falta de oportunidades socioculturales que enfrenta este grupo poblacional. La realidad presentada en los resultados de este estudio debe convertirse en oportunidad para que los tomadores de decisiones se apoyen en evidencia científica para fundamentar la revisión de las políticas públicas dirigidas a este colectivo y de esta manera impactar de manera eficiente y efectiva su calidad de vida, procurando una mayor inclusión.

\section{DECLARACIÓN SOBRE CONFLICTOS DE INTERESES}

Los autores declaran que no existen conflictos de intereses relacionados con la investigación.

\section{CONTRIBUCIÓN DE LOS AUTORES}

Primer autor: trabajo de campo, organización, análisis de información, elaboración del manuscrito. Segundo autor: trabajo de campo, organización, análisis de información, elaboración del manuscrito. Tercer autor: trabajo de campo, organización, análisis de información, elaboración del manuscrito. Cuarto autor: director del proyecto, diseño metodológico, análisis estadístico, elaboración del manuscrito.

Quinto autor: director del proyecto, diseño metodológico, análisis estadístico, elaboración del manuscrito.

\section{REFERENCIAS BIBLIOGRÁFICAS}

1. Marmot M, Bell R. Fair society, healthy lives. Public Health [revista en la internet]. 2012 [citado 28 may 2018]; 126 (Suppl. 1): 4-10. https://doi.org/10.1016/j.puhe.2012.05.014.

2. Organización Mundial de la Salud. Determinantes sociales de la salud [internet] [citado 14 jun 2017]. Disponible en: http://www.who.int/social_determinants/es/.

3. Solar O, Irwin A. A Conceptual Framework for Action on the Social Determinants of Health. Organización Mundial de la Salud [internet]; 2010 [citado 14 jun 2017]. p. 79. Disponible en: https://www.who.int/sdhconference/resources /ConceptualframeworkforactiononSDH_eng.pd $\mathrm{f}$.

4. Santos PH. Los determinantes sociales, las desigualdades en salud y las políticas, como 
temas de investigación. Rev Cubana Salud Pública [revista en la internet]. 2011 [citado 14 jun 2017]; 37(2): 136-144. Disponible en: http://scielo.sld.cu/scielo.php?script=sci_arttex t\&pid=S0864-34662011000200007.

5. García JA, Vélez C. Determinantes sociales de la salud y la calidad de vida en población adulta de Manizales, Colombia. Rev Cubana Salud Pública [revista en la internet]. 2017 Jun [citado 15 ene 2019]; 43(2): 191-203. Disponible en: http://scielo.sld.cu/scielo.php?script=sci_arttex t\&pid=S0864-34662017000200006\&Ing=es.

6. Valdez MF, Cotes KP. Desigualdades Sociales en Salud en Colombia. Informe Técnico. Sexta edición [internet]. Bogotá: Instituto Nacional de Salud, Observatorio Nacional de Salud; 2015. Disponible en: https://www.ins.gov.co/Direcciones/ONS/Resu menes\%20Ejecutivos/Resumen\%20ejecutivo\%2 Oinforme\%206\%20Desigualdades\%20sociales.p df.

7. Organización Mundial de la salud. Informe Mundial sobre la discapacidad [internet]. Ginebra: OMS; 2011 [citado 12 jun 2017]. Disponible en: http://www.who.int/disabilities/world_report/ 2011/es/.

8. Padilla MA. Discapacidad: contexto, concepto y modelos. Int Law: Rev Colomb Derecho Int [revista en la internet]. 2010 [citado 14 jun 2017]; (16): 381-414. Disponible en: http://www.scielo.org.co/pdf/ilrdi/n16/n16a12 .pdf.

9. Egea GC. Sarabia SA. Clasificaciones de la OMS sobre discapacidad [internet]. España:

Universidad de Murcia; 2001 [citado 14 feb 2016]. Disponible en https://www.um.es/discatif/METODOLOGIA/Eg ea-Sarabia_clasificaciones.pdf.

10. Organización Mundial de la Salud. Medición de la Salud y la Discapacidad.Manual para el Cuestionario de Evaluación de la Discapacidad de la OMS. WHODAS 2.0 [internet]. 2015 [citado 24 agos 2017]. p. 153. Disponible en: http://apps.who.int/iris/bitstream/handle/106 65/170500/9874573309_spa.pdf?sequence=1.

11. Puin DC, Escobar M. Actitudes frente a la vinculación laboral de personas con discapacidad. Rev Fac Med [revista en la internet]. 2002 [citado 14 jun 2017]; 50(4): 196$199 . \quad$ Disponible en: https://revistas.unal.edu.co/index.php/revfacm ed/article/view/19932.

12. Ministerio de Salud y Proteccion Social. Politica Pública Nacional de Discapacidad e Inclusion Social. 2013-2022 [internet]; 2014 [citado 21 agos 2018]. Disponible en: https://www.minsalud.gov.co/sites/rid/Lists/Bi bliotecaDigital/RIDE/DE/PS/politica-publicadiscapacidad-2013-2022.pdf.

13. Vidarte JA, Chía SL, Hormaza M, Vélez C. Determinantes sociales de la salud y discapacidad, Tunja 2012. Archivos de Medicina (Col) [internet]. 2014 [citado 21 agos 2018]; 14(1): 51-63. Disponible en: http://www.redalyc.org/articulo.oa?id=273832 164006.

14. Pérez LC. Discapacidad y exclusión social en la unión europea. Tiempo de cambio, herramientas para el cambio. Comité Español de Representantes de Personas con Discapacidad CERMI [internet]; 2003. Disponible en: http://www.cermiasturias.org/fotos/7Exclusion .pdf.

15. Gómez J, González C. Fundación Saldarriaga y Concha. Discapacidad en Colombia: Reto para la Inclusion del Capital Humano. Situación Nacional. Tomo 1 [internet]; 2008. Disponible en:

https://saldarriagaconcha.org/desarrollo.../33_ d4adc751a39b66cee8ea7b192e75cc7f.

16. Vélez C, Vidarte JA. Discapacidad y Determinantes Sociales de la Salud Estructurales e Intermedios: Diferencias por Género. Cienc Innov Salud [revista en la internet]. 2014 [citado 21 agos 2018]; 2(2): 63$69 . \quad$ Disponible en: http://publicaciones.unisimonbolivar.edu.co/rd 
igital/ojs/index.php/innovacionsalud/article/do wnload/520/502.

17. Mukaka M. Una guía para el uso apropiado del coeficiente de correlación en la investigación médica. A guide to appropriate use of Correlation coefficient in medical research. Malawi Med J [revista en la internet]. 2012 sep 24(3): $\quad$ 69-71. Disponible en: https://www.ncbi.nlm.nih.gov/pmc/articles/P MC3576830/.

18. Stang M. Las personas con discapacidad en América Latina: del reconocimiento jurídico a la desigualdad real [internet]. Santiago de Chile: Centro Latinoamericano y Caribeño de Demografía (CELADE) - División de la Población de la CEPAL; abril de 2011 [citado 20 jun 2018]. Disponible en: https://repositorio.cepal.org/bitstream/handle /11362/7135/S1100074_es.pdf.

19. Ministerio de Salud y Protección Social. Registro de Localización y Caracterización de Personas con Discapacidad - RLCPD [internet] [citado 13 dic 2013]. Disponible en: https://www.minsalud.gov.co/sites/rid/Lists/Bi bliotecaDigital/RIDE/DE/PS/Cifras\%20Registro \%20de\%20discapacidad\%20\%28Dic\%202013\%2 9.pdf.

20. Instituto Nacional de Estadistica (INE). Encuesta sobre discapacidades, autonomía personal y situaciones de dependencia [internet] [citado 22 mar 2016]. Disponible en: http://www.ine.es/dyngs/INEbase/es/operacio n.htm?c=Estadistica_C\&cid $=1254736176782 \&$ menu=resultados\&secc $=1254736194716 \& i d p=$ 1254735573175.

21. Flores $\mathrm{S}$, Jiménez AJ,Castellanos $\mathrm{W}$, Alger J, Zúñiga L, Gonzales $M$, et al. Prevalencia de discapacidad y sus características en población de 18 a 65 años de edad, Honduras, Centro América, 2013-2014. Rev Med Hondur [revista en la internet]. 2015 [citado 21 agos 2018]; 83(1,2): 7-17. Disponible en: http://pesquisa.bvsalud.org/portal/resource/pt /biblio-924.
22. Gil L, López A, Ávila C, Criollo C, Canchala A, Serrato MP. Discapacidad y su relación con variables sociodemográficas y clínicas en adultos de la ciudad de San Juan de Pasto, Nariño, Colombia. Universidad y Salud [revista en la internet]. 2016; 18(3): 505-513. Disponible en: http://dx.doi.org/10.22267/rus.161803.55

23. Daza GE, Mosquera MG. Determinantes sociales de la salud (DSS) y discapacidad en Barranquilla, 2012: resultados desde el registro para la localización y caracterización de personas con discapacidad [tesis]. Manizales: Universidad Autónoma de Manizales; 2013.

24. Fuentes GA, Sánchez H, Lera L, Cea X, Albala C. Desigualdades socioeconómicas en el proceso de discapacidad en una cohorte de adultos mayores de Santiago de Chile. Gac Sanit [revista en la internet]. 2013 [citado 21 agos 2018]; 27(3): 226-232. Disponible en: https://ac.elscdn.com/S0213911112003263/1-s2.0S0213911112003263-main.pdf?_tid=083cda6b5928-45dc-bb30-

b7ee1f396b5d\&acdnat=1534769294_2d5b040 bf8d4592ebae50e5fbd72b237

25. Departamento Administrativo Nacional de Estadística (DANE). Dirección de censos y demografía. Base de datos de registro de localización y caracterización de personas con discapacidad. Bogotá; 2010.

26. Castelblanco M, Cerquera L, Vélez C, Vidarte, JA. Caracterización de los determinantes sociales de la salud y los componentes de la discapacidad en la ciudad de Manizales, Colombia. Diversitas [revista en la internet]. 2014 [citado 21 agos 2018]; 10(1): 87-102. Disponible en: http://revistas.usta.edu.co/index.php/diversita s/article/view/1807/2215.

27. Samaniego DG. Personas con discapacidad y acceso a servicios educativos en Latinoamérica. Edit CINCA; 2008.

28. Mayer C. El derecho a la protección de la salud. Salud pública Méx [revista en la internet]. 2007 [citado 25 agos 2018]; 49(2): 144-155. Disponible en: 
http://www.scielo.org.mx/scielo.php?script=sci _arttext\&pid=S0036-36342007000200010.

29. Venturiello M. ¿Qué significa atravesar un proceso de rehabilitación? Dimensiones culturales y sociales en las experiencias de los adultos con discapacidad motriz del Gran Buenos Aires. Rev. Katálysis [revista en la internet]. 2014 [citado 21 agos 2018]; 17(2): 185-195. Disponible en: http://www.scielo.br/scielo.php?script=sci_artt ext\&pid=S1414-49802014000200185.

30. Organización Panamericana de la Salud. Discapacidad: salud de las personas con discapacidad [internet] [citado 14 abr 2016]. Disponible en: https://www.paho.org/chi/index.php?option=c om_content\&view=article\&id=82: discapacidad \&ltemid=21.

31. Pupiales B, Córdoba L. La inclusión laboral de personas con discapacidad: Un estudio etnográfico en cinco comunidades autónomas de España. Archivos de Medicina (Col) [revista en la internet]. 2016 [citado 21 agos 2018]; 16(2): 279-289. Disponible en: http://www.redalyc.org/articulo.oa?id=273849 945007.

32. Ministerio de Desarrollo Social de Chile. Caracterización General, Prevalencia Nacional de discapacidad en Chile [internet]. 2015. p. $137 . \quad$ Disponible en: https://www.senadis.gob.cl/descarga/i/3015.

33. Flores $S$, Jiménez $D$, Castellanos $W$, Alger $j$, Zúñiga I, Gonzales M, et al. Prevalencia de discapacidad y sus características en población de 18 a 65 años de edad, Honduras, Centro América, 2013-2014. Rev med hondur [internet]. 2015 [citado 2018 agosto 21]0; 83 (1): 4-17. Disponible en: http://www.bvs.hn/Honduras/UICFCM/Articulo 3_Vol83-1-Discapacidad.Sujetos.18-65a.pdf

34. Fundación Saldarriaga Concha. Informe Discapacidad Fundación Saldarriaga Concha. Día mundial de las Personas con discapacidad [internet]. Pacto de Productividad. 2012 [citado 10 nov 2016]. Disponible en: http://www.pactodeproductividad.com/pdf/inf ormediscapacidadfsc.pdf.

35. Organización Mundial de la Salud. Discapacidad y salud [internet] [citado 18 ene 2018]. Disponible en: http://www.who.int/es/newsroom/fact-sheets/detail/disability-and-health.

36. Vélez M, González A, Velásquez R. Revisión de las barreras de acceso a los servicios de salud de la población con discapacidad en Colombia entre los años 2005 a 2015. Revista CES Derecho [revista en la internet]. 2016 [citado 21 ago 2018]; 7(2): 72-83. Disponible en: http://www.scielo.org.co/pdf/cesd/v7n2/v7n2 a06.pdf.

37. López MP, Mendieta D, Muñoz MA, Díaz A, Cortés JF. Calidad de vida y discapacidad en el trastorno de ansiedad generalizada. Salud Ment [revista en la internet]. 2014 [citado 15 ene 2019]; 37(6): 509-516. Disponible en: http://www.scielo.org. $\mathrm{mx} /$ scielo.php?script=sci _arttext\&pid=S018533252014000600009\&Ing=es. 